\title{
Study on land-use around rail transit stations based on TOD theory
}

\section{Taking Wudaokou subway station in Beijing as an example}

\author{
Xin LI, Beijing Forestry University, China
}

Chunxi ZHU, Beijing Forestry University, China

Yujia ZHONG, Beijing Forestry University, China

\begin{abstract}
With the rapid development of Beijing's economy and society and the continuous expansion of urban space. In this regard, the government proposes to vigorously develop public transport, especially to increase the construction of rail transit network to solve urban transport problems. Transit-oriented development (TOD) mode is a land development mode dominated by rail transit. Through the mixed use of land, a good walking environment is established, so as to achieve the coordination of transportation and space. This paper analyses the current situation and problems of Beijing's urban rail transit development, and concludes Beijing's urban traffic problems. Based on the study of TOD mode and in-depth study of the theory of urban rail transit construction and the experience of rail transit construction at home and abroad, This paper summarizes the current situation of the traffic space, residential space, commercial space and recreational space around the west entrance of Qinghua East Road of Beijing Metro, and puts forward the redesign of the traffic space, residential space, commercial space and recreational space. I hope that the analysis of Wudaokou metro station under TOD mode can provide reference for others to do TOD theory for urban rail transit station planning.
\end{abstract}

\section{Keywords}

TOD theory, Urban transportation and land use, Rail traffic, Land layout, Beijing 


\section{Introduction}

\subsection{Background}

As China urbanization speed stepping up, a large number of urban population agglomerates. And many problems such as environmental pollution, traffic congestion and land shortage in big cities are getting worse. Facing the dual challenges of urbanization and motorization, the supply of transport infrastructure is hard to meet the growth of traffic demand. Traffic congestion is serious and the efficiency of the urban land utilization is low. The contraction of urban land use and urban traffic is getting more and more prominent. More and more scholars have paid attention to the development of the urban land use and transport system interaction research, probing into the rational development of land resources and transportation system of the benign interaction, so as to realize the coordinated development of urban economy and society. As a large-scale and large-volume transportation mode, rail transit can not only solve traffic congestion and the accompanying environmental pollution problems, but also lay a foundation for Beijing to build a "two-axis - two-belt-multi-centre" urban spatial structure. ${ }^{[1]}$ This paper analysed the implementation experience and inspiration on the basis of theoretical studies of many scholars at home and abroad. It also discussed the influence of TOD strategy on urban spatial structure by analysing successful TOD cases. The general method and mode research of land development around urban rail transit stations under the TOD mode has also been considered.

\subsection{Application situation}

\subsubsection{TOD mode construction in Arlington County, USA}

The R-B corridor in Arlington County was constructed on the basis of intercity rail. It begins from Ballston station, along the site of Wilson Avenue, Court House, Clarendon and Virginia Square, then finally ends at Rosslyn station. R-B corridor emphasizes integrating land exploitation and integrated rail transit construction. It actively encourages concentrating residential, office and retail development near the station, thus the residents can easily use urban rail to travel. Meanwhile, it designs a pedestrian-friendly walking environment. It also combines the public transport system with complete pedestrian and bicycle facilities, which efforts to build a good community environment. ${ }^{[2]}$

The success of R-B corridor can be summarized in the following three aspects: formulating reasonable strategic planning, reasonable land use planning, reasonable land development and transportation design. In the implementation of TOD mode, R-B corridor not only vigorously develops rail transit, but also attaches great importance to the construction of conventional public transportation system and walking and cycling system. The organic integration of these different transportation modes for public transportation makes R-B corridor the most popular place for living, working, traveling and leisure.

\subsubsection{TOD mode in Stockholm}

Stockholm, the capital of Sweden, is a low-density city with the population of less than 800,000 . More than 90 percent of Swedish residential units have been built since 1946, but Sweden is also one of the richest countries in Europe, with a GDP per capita of $\$ 17,900$ in 1990 and the highest car ownership rate in Europe, at 420 cars per 1,000 people. It is worth noting that although Stockholm is fully equipped with the prerequisites for the full development of cars, due to the use of public transport-oriented land use mode, it has well controlled the use of vehicles and has become the most public transport-oriented city in Europe. ${ }^{[3]}$

Half of Stockholm's population lives in the central city and the other half in the satellite city. All these satellite cities built in postwar are located at radiate railway station, which scale and layout for pedestrian traffic: railway station entrances are incorporated with the public square. Surrounding supermarkets, 
stores, day care centers and other facilities, and configuration of the seats, the newsstand, roadside cafes, walks covered nest and flower beds and other facilities walkways connecting with the surrounding residential area. The density of buildings from the center to the periphery has been gradually reduced and the level of buildings has been gradually improved, which is especially conducive for low-income residents to use of public transport.

\subsubsection{TOD mode in Beijing Yizhuang Light Rail}

Yizhuang Light Rail is a light rail line connecting the downtown area and Yizhuang New Town. In the planning of LZ line, TOD development and construction are carried out for the stations along and around the line. According to TOD principle, LZ stations are distributed as far as possible to make it convenient for people to travel in residential or commercial areas. It also lengthens the distance in industrial areas to increase the speed thus ensure the transportation capacity. On the main streets such as Ronghua Road, the distance between LZ stations should be kept at about 800 meters to assure convenient travel of citizens within a radius of 400 meters, and to boost the development potential of the land along the road, so as to form a continuous dense urban development belt along the street. The distance between LZ stations in the industrial zone can be extended to about $1.5-3 \mathrm{~km}$. According to TOD principle, a total of 12 stations are set along LZ. Within a 10-minute walking distance of about 500 meters around the light rail station, it mainly arranges the land for schools, middle and high-end residences, commerce and retail, etc., to ensure the convenience and accessibility of public travel. The density is controlled at 2-3, and the maximum is 4 , with an average of 2.5. Within a range of 500-1000 meters around the light rail station, it can be connected to the light rail station by motor vehicles. Villas, independent houses, townhouses and some ordinary houses are mainly arranged, with a density of 1-2. ${ }^{[4]}$

\section{Method}

\subsection{Introduction of TOD mode}

"TOD" development mode aims to optimize the community environment, improve the quality of life, and promote the use of public transportation. It emphasizes the compact development around public transportation stations, and establishes a good walking environment through the mixed use of land, so as to realize the coordination between traffic and space. ${ }^{[5]}$

The characteristics of TOD mode are: Compact layout and mixed use of land near bus stops; Providing good public transport facilities and services; Land development is based on the principle of improving the use of public transportation; Public facilities and public Spaces are adjacent to bus stops; The public station becomes the transportation hub within the region; Convenient blocks provide a good environment for walking and cycling.

By summarizing the TOD planning principles proposed by Peter Calthorpe and the 3D principles proposed by Cervero and Kockelman ${ }^{[6]}$, this paper summarizes the TOD planning principles as follow: Compact urban structure should be selected for regional urban development to make the regional growth structure consistent with the development direction of public transportation; Adopt mixed-land development, bike-friendly and pedestrian-friendly planning principles to replace the ubiquitous singleuse regional planning control principles, creating a good walkable and bicycling traffic environment; Urban micro-design should be oriented towards the public sector and human scale rather than the private sector and car scale; Priority should be given to the development of public transport. Bus stations are close to the community and become the hub of the region, with efficient public transport service facilities. 
A typical TOD mode is mainly composed of the following functional structures: core business district, office district, residential district, sub-district, public space, etc. According to the different location, characteristics and functions of "TOD", "TOD" can be divided into two types, namely "urban TOD" and "community TOD".

1)"Community TOD" mainly provides diversified housing with high residential density, and provides entertainment, catering, retail and municipal public facilities and other community services to nearby residents.

2)"Urban TOD" area is located on the main line of the regional public transport network, such as the light rail station or bus station, and becomes the commercial center or job center. Such area has a high density of land development and is relatively large..$^{[7]}$

\subsection{Features of TOD mode}

\subsubsection{Spatial scale of TOD mode}

According to the study of TOD spatial scale in some American cities, it is found that the comfortable walking distance for most people is within 150 meters, $40 \%$ of people are willing to walk $300 \mathrm{~m}$, but only less than $10 \%$ are willing to walk 800 meters. In foreign countries, a distance of 5-15 minutes on foot is usually adopted, with a radius of about 400-800 meters as the space range of TOD. At the same time, the study also found that the comfortable walking distance is also affected by the terrain, climate, traffic network and other factors in different regions. So, the scale and size of TOD in different regions differ from different environmental conditions. ${ }^{[8]}$ In the application of TOD mode in Beijing, a comprehensive analysis should be conducted to determine the reasonable spatial scale of TOD according to the spatial structure characteristics, economic and social conditions and travelers' preferences of Beijing.

When dividing Beijing urban rail transit stations surrounding land, regions with rail transit stations as the core are roughly within a circle of $600 \mathrm{~m}$, based on the relation of TOD spatial scale.

\subsubsection{The density of TOD mode}

Relevant studies abroad mainly focus on the critical low density of TOD mode. The research community generally believes that high-density development is more likely to bring success to the TOD mode and can improve the utilization rate of public transport, but there is less discussion on the traffic congestion caused by excessive land development. Compared with the low density and car-oriented characteristics of European and American countries, the population density of large and medium-sized cities in China is generally high. Therefore, How to study the reasonable TOD structure and density based on the current traffic supply and demand in large and medium cities in the country is more important.

\subsubsection{Land use characteristics of TOD mode}

TOD mode advocates mixed-use land development, which distributes shops, houses, parks, offices and public building facilities within walking distance of bus stops. So that the community residents and workers can solve the shopping, entertainment and other travel in the community, thus reduce unnecessary travel.

\subsection{Rail transit development actuality in Beijing}

In the 21st century, Beijing has strengthened and established the priority and primary role of public transportation, especially urban rail transit, in the comprehensive transportation system of the urban center. Taking rail transit as an important measure to improve the overall function of the city. During the 11th Five-Year Plan period, Beijing's subway operating mileage has increased more than twofold from 
$114 \mathrm{~km}$ at the end of 2005 to $336 \mathrm{~km}$ in 2009 . The average daily passenger volume has increased from 1.86 million in 2005 to more than 6 million in 2009, and rail transit has gradually become the main mode of transportation in the urban area. ${ }^{[9]}$ At the same time, in order to deal with the serious traffic congestion problem, Beijing proposes to raise 500 billion yuan by 2020 to expand Beijing's rail transit lines to about $1000 \mathrm{~km}$, so as to achieve the goal of covering the central city with rail transit.

\subsection{Urban traffic problems in Beijing}

\subsubsection{The agglomeration of the central city leads to excessive concentration of traffic flow}

In recent years, Beijing has witnessed rapid economic development, speedy increase of urban population, high-density development of central city and continuous expansion of urban scale. As the unordered sprawl of urban has not been effectively curbed, the non-scientific evolution from non-construction land to construction land is on the rise. In addition to Beijing's own urban structure characteristics and road construction, Beijing has gradually formed a "single center + loop" urban structure. Its concrete phenomenon is the population and industry gather to city center. The high-density exploitation is mainly centralized within the Fifth Ring Road. The residential population is 7.87 million, accounting for $63 \%$ of the total residential population of Beijing. On the one hand, the development of high-density land in the city center makes the protection of cultural relics passive; on the other hand, it also brings a series of traffic problems in Beijing.

\subsubsection{The shortage of land resources brings difficulties to the construction of transportation}

China is short of land resources, the resources for road traffic construction is very limited. At present, the per capita urban road area is only 3.39 square meters, and the urban road area ratio is only $12 \%$, compared with about $30 \%$ in developed countries. ${ }^{[10]}$ Therefore, the capacity of road network cannot meet the traffic demand. It is difficult to meet the needs of urban traffic development through massive investment and rapid extension of roads. The only way for the sustainable development of Beijing's transportation is to implement the compact land use mode and strengthen the development of public transportation, especially the construction of rail transit with large capacity.

\subsubsection{Motorization is speeding up, and traffic energy consumption is intensifying.}

At present, the number of motor vehicles in Beijing increases by $10 \%$ every year. By the end of 2007 , the number of motor vehicles in Beijing had exceeded 3 million. ${ }^{[11]}$ This put forward higher requirement to traffic, environment and energy. Although the urban road network of Beijing has basically formed a grid, loop and radiation combined layout, the speed of road construction is far from keeping pace with the growth momentum of traffic demand. The rapid development of private vehicles not only intensifies traffic energy consumption but also brings a certain burden to Beijing's environment.

\subsubsection{The block scale is too extensive, and the pedestrian system is deficient.}

The distance between the main roads in Beijing is 700 to 1200 meters, which is the urban form of "large blocks with sparse grids". ${ }^{[12]}$ The large block scale is not conducive to provide a safe and comfortable walking environment, and people have fewer options for walking. Both motor vehicles and pedestrians are concentrated on the urban arterial roads. In addition, large-scale blocks also require a relatively large traffic capacity in the scale of the trunk road, leading to a large section of the road, which is not suitable for pedestrians to cross freely. For example, Beijing's main roads measure more than 50 meters, and some sections of the ring road can even reach 100 meters. The general branch will also be around 20 to 30 meters. 
To sum up, the implementation of TOD mode in Beijing urban rail transit is necessary and applicable, and the implementation of TOD mode in Beijing rail transit will be conducive to the development of the city and the use of citizens.

\subsection{The research site}

Wudaokou Station (No. 1304 station) along Beijing Metro Line 13 was completed and opened to traffic in September 2002. Line 13 ends at Xizhimen and Dongzhimen stations. Wudaokou station is a midway on Line 13. There are two entrances and exits: North Exit $A$ and South Exit B. Due to the railway environment near Wudaokou Subway station, the nature of the surrounding land and the condition of its own transportation hub, traffic congestion of the roads around Wudaokou subway is severe, which causes great inconvenience for citizens' life and travel.

In the current situation investigation, this paper mainly adopts two methods: field investigation analysis and cartographic research. The field investigation and analysis mainly includes subjective experience recording. Cartographic research includes two processes: making analysis chart and marking information according to field investigation. In the analysis of the investigation results, this paper first analyzes the required characteristics of the space elements under TOD mode, and then finds out the unreasonable practices of the current space from the field investigation data.

According to TOD spatial scale principle and the investigation situation, Wudaokou Subway station surroundings in the investigation is an area which core is the station and is covered with a radius of about 600 meters. In the investigation, the land is mainly residential land, including Huaqing jiayuan, No. 20 Chengfu road, Chengjiansi communities, Dongwangzhuang community, Xiwangzhuang community and Beijing language and culture university family area. Commercial land is distributed on the east and west sides of the subway station, and there are three office land in the site, which is not immediately adjacent to the subway station. Besides the subway, there are also railway land along Line 13 . There is protective green land around the transportation facilities land, two primary schools are near the residential area.

\section{Results}

\subsection{Existing problems}

\subsubsection{Traffic space elements}

1) Walking system

Walking accessibility is a necessary condition to achieve TOD mode, which is directly related to the quality of TOD community.

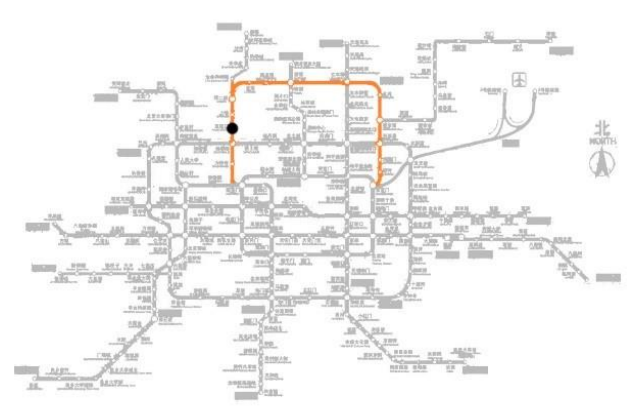

Picture1: Line 13 and the location of Wudaokou railway station 


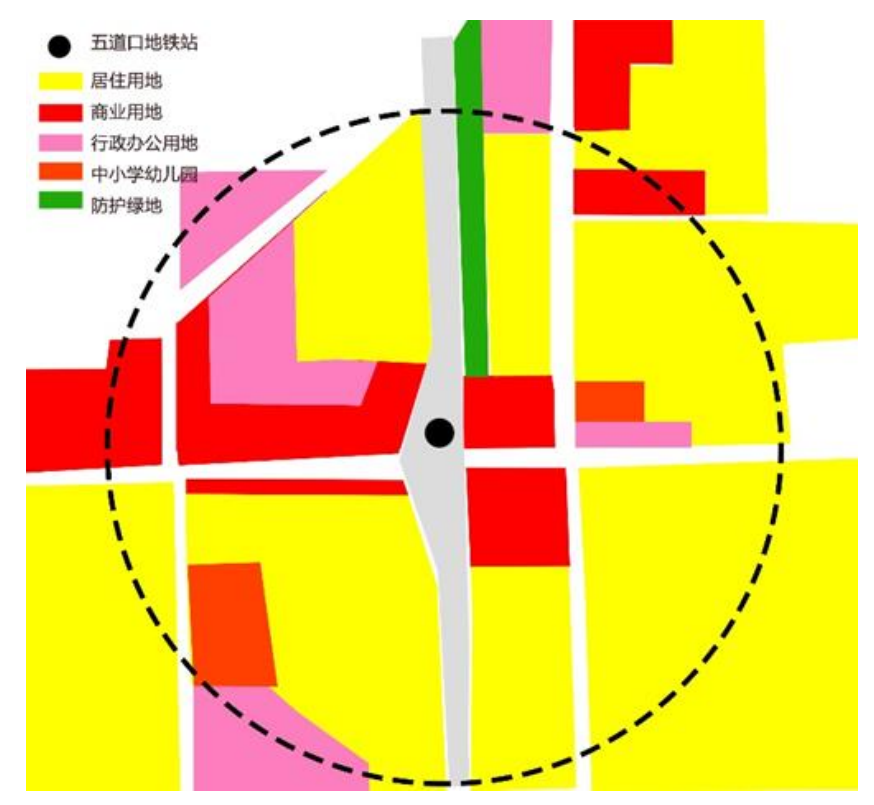

Picture2: Wudaokou railway station and surrounding land use

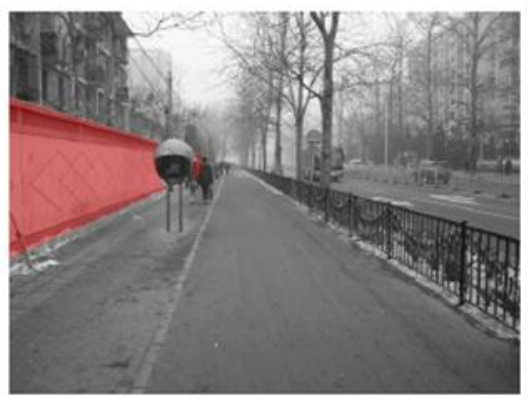

Picture 3:Chengfu Road
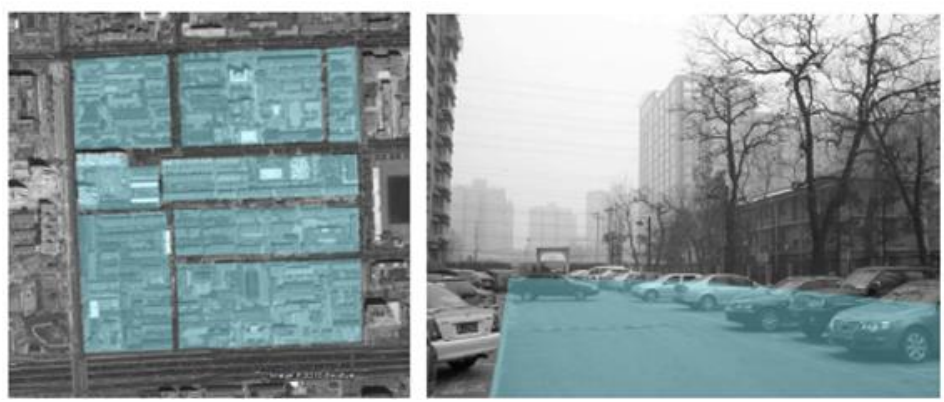

Picture 4: China University Of Geosciences path

The road enclosure interface is monotonous. The urban pedestrian way of Wudaokou station (on both sides of Chengfu road east) is adjacent to China University Of Geosciences and Beijing Language \& Culture University, and its enclosing interface is a tall and long wall erected along the street side, which leads to the deterioration of the walking environment quality and only bears the walking function negatively.

The road grid in the community is too large. As a result, there are fewer pedestrian paths for people to choose from, and they are relatively concentrated in several main community paths, which forms a space within the community that no one goes to (because it is often replaced by parking lots), resulting in the decline of the walking environment quality.

2) Bicycle system

There is no continuity of cycle lanes on important paths. On important traffic paths, bicycle lanes are occupied by motor vehicles. Drivers take bicycle lanes as free parking spaces and park their cars on motorized roads for a long time. 


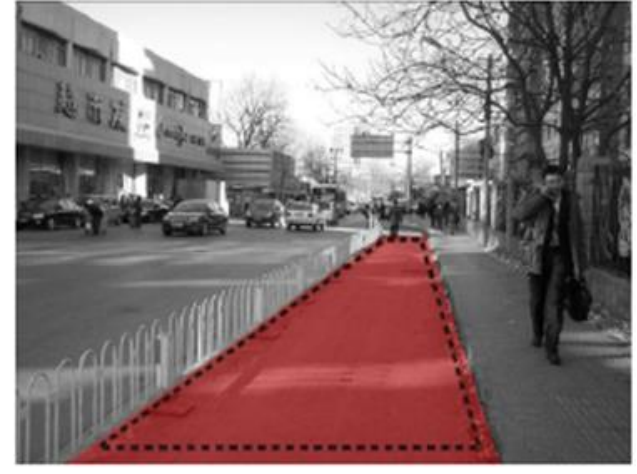

Picture 5:Vichels on Chengfu Road

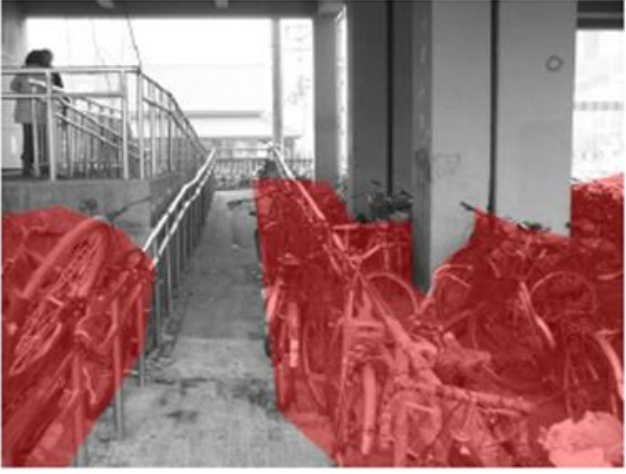

Picture 6:Small capacity of Wudaokou railway station

3) Motor vehicle system

The width of Heqing Road is close to the urban ring road. However, due to the strong directionality of its traffic flow, in different peak periods, only one direction is blocked while the other direction is empty, resulting in the waste of road resources and the destruction of urban space.

\subsubsection{Residential space elements}

Unit residential area is a closed area, which does not allow outside vehicles and pedestrians to cross. Additionally, it is large in scale and often occupies the whole block independently, which will directly affect the realization of regional TOD mode.

In many groups, the residential form is just a slab building followed by another slab building. There is not enough green open space in the groups, and the spare space along both sides of the road is occupied by vehicles.

The enclosed courtyard wall along Chengfu Road has a great negative effect on urban pedestrian space and environmental quality. Communities connects the road directly with tall concrete walls which extend for hundreds of meters, depriving walkways of functional architectural interface enclosure and reducing the attractiveness of the walking environment.

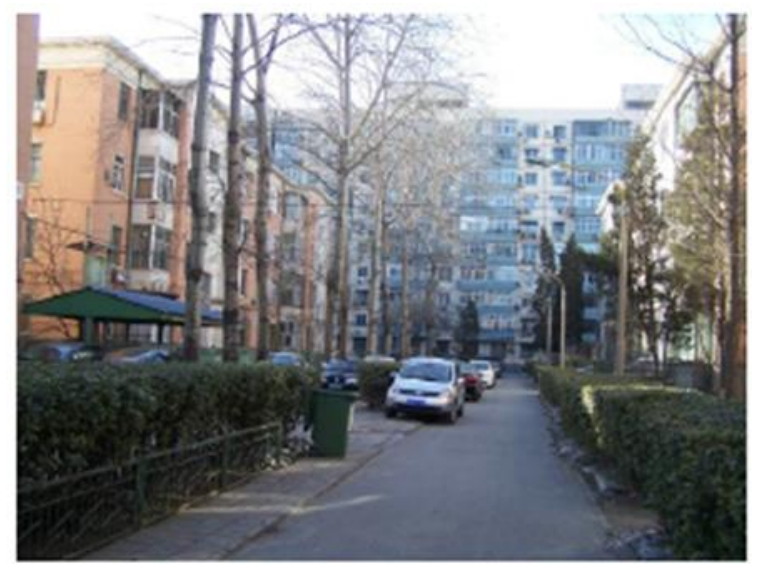

Pitcture 7: Dongwangzhuang community

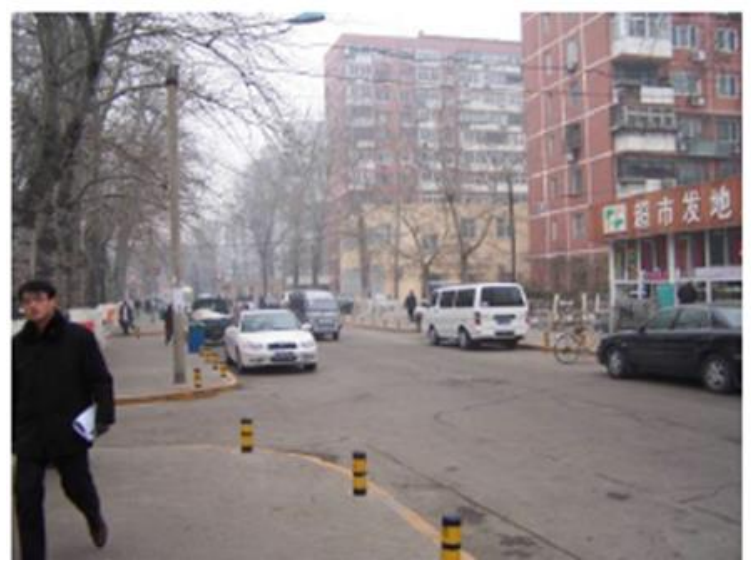

Picture 8: Beijing Language \& Culture University

\subsubsection{Commercial space elements}

The connection between Wudaokou Hualian Mall and Wudaokou Subway station is shoddy. Although Hualian Mall next to Wudaokou Light Rail station is only about 50 meters away from the entrance and exit, they were separated by a train track. It often causes a large number of people to jam and wait for 
the train to pass. At the same time, the walking path environment between the two is not properly designed, resulting in the uncomfortable walking process from the station to the mall.
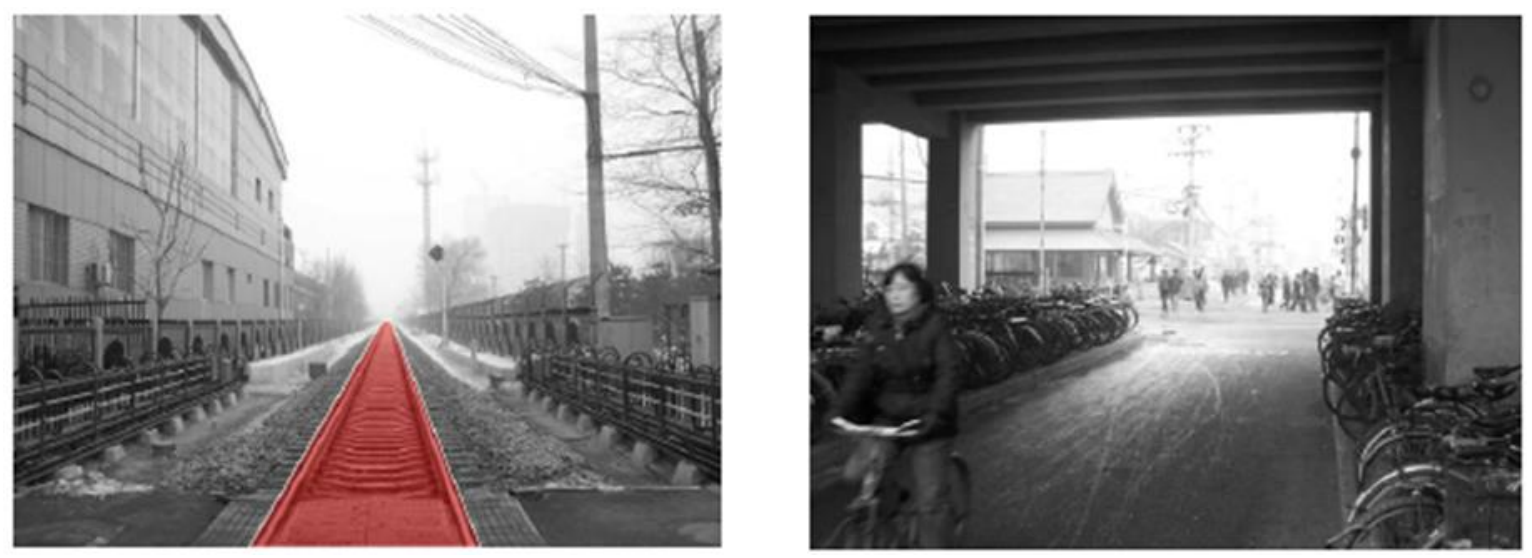

Picture 9, 10: The connection between Wudaokou and Hualian Mall is shoddy

\subsubsection{Recreation space elements}

The corner space around Wudaokou cinema is not utilized. It is currently used as a fee-paying parking lot and is full of motor vehicles.

\subsection{Suggestions for improvement}

In order to make Wudaokou Subway station area conform to the characteristics of TOD mode, promoting the development of surrounding areas effectively and facilitate citizens to use, the following four aspects are redesigned.

\subsubsection{Traffic space redesign}

1) Ensure the continuity of the walkway

Pedestrian walkways should be provided on both sides of urban roads so that the sidewalks can become continuous between blocks. The center of the walkway should not be hindered by

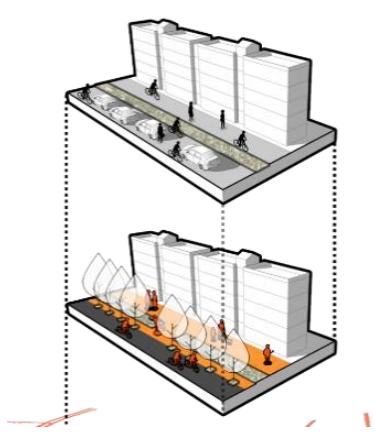

Picture 11: Redesign of padestrian paths and bicycle lanes green planting and environmental facilities. Greening should be planted on both sides of the walkway as far as possible.

2) Independence of bike lanes

A green separation belt of appropriate width should be applied between the bicycle lane and the motor vehicle lane. The best form of green belt is a 2-5 meters wide patch composed by grass, low shrubs and tall trees.

3) Rest facilities at bus stops

The bus station and the surrounding environment are redesigned to provide a favorable waiting environment. It would attract more citizens to choose public transport thus relieve the traffic pressure. 


\subsubsection{The living space redesign}
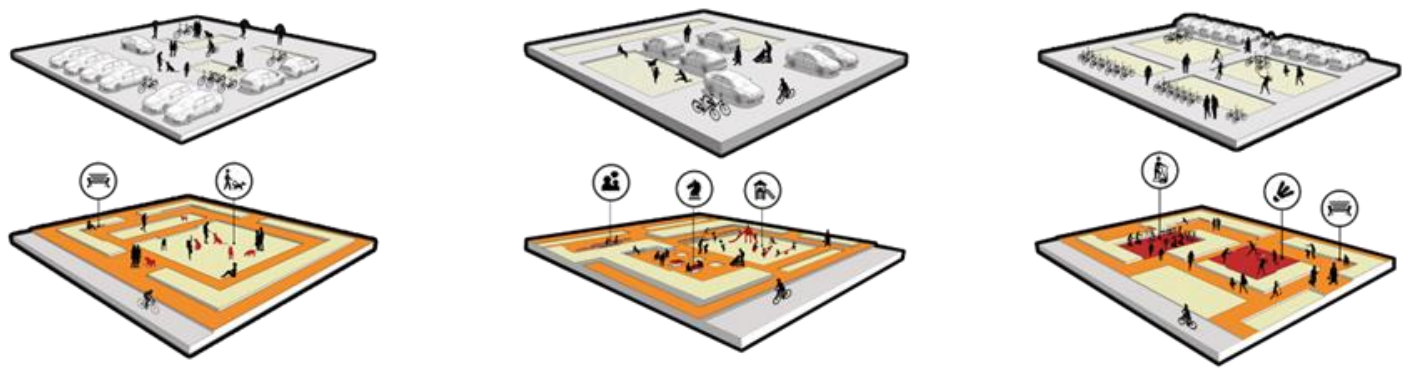

Picture 12: Redesign of the front square and roads in the residential area

Redesign the front square and the disorderly spatial structure of the residential square and road. Put forward concrete solving strategy for several kinds of problems. The broken original site was redesigned during the planning. Active spaces such as dog walks and recreation facilities are introduced into the space. Besides, parking lots are arranged reasonably, and the recreation facilities for children, chess and place to rest. It is planned to increase outdoor fitness facilities, recreation facilities and sports stadium where is lack of activity space now.

\subsubsection{The commercial space redesign}

At present, the connection between the commercial district and the subway station is mainly shopping pedestrian path. The pedestrian path space could be appropriately enlarged to serve as the connection square combining commercial center at the railway station.

The present residential area along the road street is transformed into the ground floor business, and there are more continuous commercial storefronts on both sides of the main road leading to the residential area.

\subsubsection{Recreation space redesign}
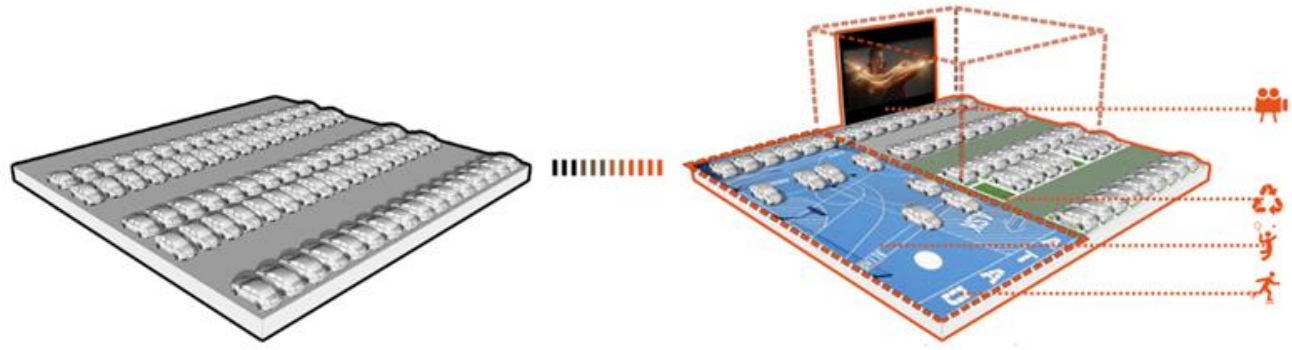

Picture 13: Redesign of the corner space

The corner plaza around Wudaokou Cinema is no longer a single motor vehicle parking lot. The corner space would become a parking lot that can not only meet the parking demand, but also provide people with activities during the time of parking cars. Greening is planted in the site to improve the existing environment. The temporary activity space can have many functions, such as sports, rest, and drive-in theater. The function of the space puts people in the first place, rather than merely meeting the parking needs of motor vehicles. 


\section{Conclusions}

In order to realize the maximum utilization of urban land and environmental resources in China, the transportation system and land use must be considered comprehensively. TOD mode is the perfect interpretation of the coordination between transportation system and land use development. This paper discusses such urban problems from the perspective of TOD mode introduction, cases study, current situation of Beijing rail transit, current situation around Wudaokou Subway station and the redesign of surrounding space elements. Based on the examples around Wudaokou Subway station, this paper points out the problems that should be paid attention to in the development of TOD mode around the rail transit station. Meanwhile, it redesigns the mixed land use in the micro-TOD community from the perspective of reality, which is an enlightenment to the practical implementation.

\section{References}

[1] Yongxin Quan, Jifu Guo, Jizhen Guan, Lei Yu, Huimin Wen, Y. (2007) Research on Traffic Congestion Evaluation and Empirical Analysis of Beijing. $1^{\text {st }} \mathrm{edn}$.

[2] Dong Li, D. (2003) 'New urbanism', Beijing planning review, 6, 90-92.

[3] Xiao Wu, X. (2008) 'Planning and Construction of New Town of Postwar Stockholm and Its Enlightenments', Huazhong Architecture, 26, 164-170.

[4] Yulin Jiang, Y. (2009) Transit-Oriented Development the Concept and Its Practice in China. $1^{\text {st }}$ edn. Beijing: China Communications Press.

[5] Earl G Bossard, Jeff Hobbs, E. (2002) 'Envisioning Neighbourhoods with Transit-Oriented Development Potential', The Mineta Transportation Institute, College of Business, State University,83-92.

[6] Statewide Transit-Oriented Development Study . Factors for Success in California-Final Report

[7] Chuncai Wang, C. (2007) 'Study on the interaction mechanism of urban transport and urban spatial evolution', Beijing Jiaotong University.

[8] Xiaoliang Zhang, X. (2007) 'Study on the Planning of Traffic Join and Cooperation for TOD Railway', Huazhong University of Science Technology.

[9] Daqian Tang, D. (2008) 'Study on Transit Oriented TOD Town', Tianjin University.

[10] Weiming Zhou, W. (2010) 'The Study on the Land Use Surrounding Urban Mass Transit Stations Based on Transit-Oriented Development', South China University of Technology.

[11] Available at: https://www.bjsubway.com/

[12] Wei Wang, W. (2001) Study on the theory system of sustainable development of urban transportation system. $1^{\text {st }}$ edn. Beijing: Science Press.

[13] Lingbing Kong, L. (2009) 'Urban Transportation Development in China: Evolving Environment vs. Sustainable Development in a New Situation', Urban Transport of China, 7(6), 8-16.

[14] Lei Fu, L. (2009) 'Studied on the TOD mode in Urban Mass Rail Transit Station Areas', South jiaotong University.

[15] Yanying Wang, Yu Huang, Y. (2016) 'Index System of Traffic Congestion Evaluation in Beijing Based on Big Data', Journal of Transportation Systems Engineering and Information Technology,4, 231-240.

[16] Xiaoping Yu, X. (2016) 'Synergetic Development of Urban Rail Transit System and Polycentric Metropolitan', Beijing jiaotong University. 
[17] 北京机动车数量近 400 万辆[J]. 道路交通管理,2009,11:41.

[18] Chen Yu, Min Niu, Saina Xu, C.(2020) 'Research on the Development Mode of TOD Project -- A case study of Songling Avenue Comprehensive Transportation Hub in Wujiang District', Intelligent City, 15,117118.

[19] Quan Liu, Yiting Shi, Q. (2020) 'Integrated Intensity Regulation in TOD Planning Along Rail Transit Line', Urban Planning International, 35(04), 131-137.

[20] Yu Xi, Y. (2020) 'Research on comprehensive transportation Hub Planning of Small and Medium-sized cities based on TOD mode', City\& House, 27(06), 171-172. 